\title{
Complex Chebyshev Polynomials on Circular Sectors With Degree Six or Less
}

\author{
By U. Grothkopf and G. Opfer
}

\begin{abstract}
Let $T_{n}^{\alpha}$ denote the $n$th Chebyshev polynomial on the circular sector $S^{\alpha}=\{z$ : $|z| \leqslant 1,|\arg z| \leqslant \alpha\}$. This paper contains numerical values of $\left\|T_{n}^{\alpha}\right\|_{\infty}$ and the corresponding coefficients of $T_{n}^{\alpha}$ for $n=1(1) 6$ and $\alpha=0^{\circ}\left(5^{\circ}\right) 180^{\circ}$. Also all critical angles for $T_{n}^{\alpha}, n=1(1) 6$ are listed, where an angle is called critical when the number of absolute maxima of $\left|T_{n}^{\alpha}\right|$ changes at that angle. All figures are given to six places. The positions (and hence the number) of extremal points of $T_{n}^{\alpha}, n=1(1) 6$ are presented graphically. The method consists of a combination of semi-infinite linear programming, finite linear programming, and Newton's method.
\end{abstract}

1. Introduction. If $D$ is any nonempty compact set in $\mathbf{C}$ and $n \in \mathbf{N}$, then the polynomial

$$
T_{n}(z)=z^{n}+a_{n-1}^{(n)} z^{n-1}+a_{n-2}^{(n)} z^{n-2}+\cdots+a_{0}^{(n)}
$$

which is uniquely defined by

$$
\begin{aligned}
\left\|T_{n}\right\|_{\infty} \leqslant \| z^{n}+b_{n-1} z^{n-1}+b_{n-2} z^{n-2}+ & \cdots+b_{0} \|_{\infty}, \\
& \text { for all } b_{0}, b_{1}, \ldots, b_{n-1} \in \mathbf{C},
\end{aligned}
$$

is called the $n$th Chebyshev polynomial (or T-polynomial) with respect to D. The norm \|\|$_{\infty}$ is the ordinary uniform norm in $C(D)$ over $\mathbf{C}$. We will be concerned with $T$-polynomials with respect to circular sectors

$$
S^{\alpha}=\{z \in \mathbf{C}:|z| \leqslant 1,|\arg z| \leqslant \alpha\}, \quad \alpha \in[0, \pi] .
$$

Circular sectors are convenient for subdividing the plane. There is an investigation by Coleman and Monaghan [2] in which certain complex Bessel functions are approximated on circular sectors. Another idea of approximating complex Bessel functions on circular sectors is presented by Elliott [3].

$T$-polynomials with respect to $S^{\alpha}$ will be denoted by $T_{n}^{\alpha}$. Properties of $T_{n}^{\alpha}$ are summarized in

THEOREM 1.1. (1) $m \geqslant n \Rightarrow\left\|T_{m}^{\alpha}\right\|_{\infty} \leqslant\left\|T_{n}^{\alpha}\right\|_{\infty}$ for all $\alpha \in[0, \pi]$.

(2) $0 \leqslant \alpha \leqslant \beta \leqslant \pi \Rightarrow\left\|T_{n}^{\alpha}\right\|_{\infty} \leqslant\left\|T_{n}^{\beta}\right\|_{\infty}$ for all $n \in \mathbf{N}$.

(3) $\left\|T_{n}^{\alpha}\right\|_{\infty} \leqslant 1$, where equality occurs if and only if $T_{n}^{\alpha}=z^{n}$.

(4) The coefficients $a_{j}^{(n)}, j=0,1, \ldots, n-1$, of $T_{n}^{\alpha}$ are all real.

(5) $T_{n}^{\alpha}(z)=z^{n} \Leftrightarrow \alpha \geqslant n \pi /(n+1)$.

Proof. Geiger and Opfer [4].

Received October 8, 1981; revised January 4, 1982.

1980 Mathematics Subject Classification. Primary 41A10, 41A50, 65A05, 65E05. 
In that paper one also finds $T_{1}^{\alpha}$ explicitly for all $\alpha \in[0, \pi], T_{2}^{\alpha}$ for all $\alpha \geqslant 15^{\circ}$, and $T_{3}^{\alpha}$ for $35^{\circ} \leqslant \alpha \leqslant 60^{\circ}$. Numerical results for $T_{n}^{\alpha}, n=1,2,3,4$, and $\alpha=0^{\circ}\left(10^{\circ}\right) 90^{\circ}$, are given by Opfer [9]. That paper also contains numerical expressions for certain $T$-polynomials with respect to squares and rectangles.

It should be stressed that by (5) of Theorem 1.1 all $T$-polynomials $T_{n}^{\alpha}$ are known when $\alpha \geqslant n \pi /(n+1)$. Therefore the following tables do not contain entries for $\alpha>n \pi /(n+1)$. In Table 1 we present explicit expressions for some selected $T$-polynomials $T_{n}^{\alpha}, n=1,2,3$, which are computed from the expressions given by Geiger and Opfer [4]. As is well known, the ordinary $T$-polynomials $T_{n}^{0}$ obey a three-term recurrence relation which takes the form

$$
T_{n}^{0}(z)=(z-1 / 2) T_{n-1}^{0}(z)-(1 / 16) T_{n-2}^{0}(z), \quad n=3,4, \ldots,
$$

(Abramowitz and Stegun [1, Formula 22.7.8]). From the explicit expressions for $T_{j}^{\pi / 3}, j=1,2,3$, given by Geiger and Opfer [4], one finds

$$
T_{3}^{\pi / 3}(z)=(z-1 / 2) T_{2}^{\pi / 3}(z)-(\sqrt{3} / 2-1) T_{1}^{\pi / 3}(z), \quad z \in S^{\pi / 3} .
$$

However, this formula does not carry over to larger $n$, which can be seen by inserting $z=0$. Thus, as could be expected, the complex $T$-polynomials in general do not follow a three-term recurrence relation.

\section{TABLE 1}

Explicit expressions for coefficients in $T_{n}^{\alpha}(z)=z^{n}+a_{n-1}^{(n)} z^{n-1}$ $+a_{n-2}^{(n)} z^{n-2}+\cdots+a_{0}^{(n)}, n=1,2,3 ; \alpha=0^{\circ}, 30^{\circ}, 45^{\circ}, 60^{\circ}, 90^{\circ}$

\begin{tabular}{|c|c||c|c||c|c|c|}
\hline$\alpha$ & $a_{0}^{(1)}$ & $a_{0}^{(2)}$ & $a_{1}^{(2)}$ & $a_{0}^{(3)}$ & $a_{1}^{(3)}$ & $a_{2}^{(3)}$ \\
\hline $0^{\circ}$ & $-1 / 2$ & $1 / 8$ & -1 & $-1 / 32$ & $9 / 16$ & $-3 / 2$ \\
\hline $30^{\circ}$ & $-\sqrt{3} / 3$ & $1 / 3$ & $-2 \sqrt{3} / 3$ & & & \\
\hline $45^{\circ}$ & $-\sqrt{2} / 2$ & $\sqrt{2}-1$ & -1 & $\begin{array}{c}-2(8-3 \sqrt{2} / 2 \\
-\sqrt{2}+2(5-2 \sqrt{2})) / 17\end{array}$ & $\begin{array}{c}-a_{0}^{(3)}(\sqrt{2}+3) / 2 \\
+\sqrt{2} / 2\end{array}$ & $\begin{array}{c}a_{0}^{(3)}(\sqrt{2}-1) / 2 \\
-\sqrt{2} / 2-1\end{array}$ \\
\hline $60^{\circ}$ & $-1 / 2$ & $1 / 2$ & -1 & $(\sqrt{3}-3) / 4$ & $(4-\sqrt{3}) / 2$ & $-3 / 2$ \\
\hline $90^{\circ}$ & 0 & $\sqrt{2}-1$ & $\sqrt{2}-2$ & & & \\
\hline
\end{tabular}

Associated with each $T$-polynomial is the set of extremal points

$$
E_{n}^{\alpha}=\left\{z \in S^{\alpha}:\left|T_{n}^{\alpha}(z)\right|=\left\|T_{n}^{\alpha}\right\|_{\infty}\right\},
$$

which is also referred to as set of critical points. Since polynomials are holomorphic functions, the set $E_{n}^{\alpha}$ is a nonempty compact subset of the boundary $\partial S^{\alpha}$ of $S^{\alpha}$. The symmetry of the $T$-polynomials (i.e., $T(\bar{z})=\overline{T(z)}$ ) implies that $z \in E_{n}^{\alpha} \Rightarrow \bar{z} \in E_{n}^{\alpha}$. Therefore it is reasonable to introduce the essential set of critical points by

$$
\text { ess } E_{n}^{\alpha}=\left\{z \in E_{n}^{\alpha}: \operatorname{Im} z \geqslant 0\right\} \text {. }
$$


Definition 1.1. Let $n \in \mathbf{N}$ be fixed. We call $\alpha \in[0, \pi]$ a critical angle of the corresponding $T$-polynomial $T_{n}^{\alpha}$ if there is an $\varepsilon_{0}>0$ such that

$$
\text { \# ess } E_{n}^{\alpha} \neq \# \text { ess } E_{n}^{\alpha+\varepsilon} \text { or \# ess } E_{n}^{\alpha-\varepsilon} \neq \# \text { ess } E_{n}^{\alpha}
$$

for all $\varepsilon$ with $0<\varepsilon<\varepsilon_{0}$. The set of all critical angles will be denoted by $A_{n}$.

From Theorem 1.1, part (5), we know that the largest value in $A_{n}$ always is $\alpha=n \pi /(n+1)$, since for $\alpha \geqslant n \pi /(n+1)$ the number of extremal points becomes infinite, whereas for $\alpha<n \pi /(n+1)$ this number turns out to be finite. At the end of Section 2 we shall see that $0 \notin A_{n}$, thus $A_{n}$ is a subset of $] 0, \pi[$. We may assume that the critical angles are ordered. To mention some examples

$$
\begin{gathered}
A_{1}=\left\{45^{\circ}, 90^{\circ}\right\}, \\
A_{2}=\left\{\sim 12^{\circ}, 45^{\circ}, 72^{\circ}, 120^{\circ}\right\},
\end{gathered}
$$

where $\sim 12^{\circ}$ indicates that this value is close to $12^{\circ}$ but not precisely known (Geiger and Opfer [4]). A more precise value is given in Table 8.

We shall see later that the critical angles can be detected and computed by the use of the corresponding dual program. If, however, two extremal points coalesce or one extremal point splits at $z=0$ or $z=1$ for a certain angle $\alpha$, then this angle (which is not critical according to our definition) cannot be detected by this method.

If

$$
A_{n}=\left\{\alpha_{1}^{(n)}, \alpha_{2}^{(n)}, \ldots, \alpha_{k_{n}}^{(n)}=n \pi /(n+1)\right\}
$$

then, by the definition of the critical angles, the numbers

$$
\left.q_{j}^{(n)}=\# \operatorname{ess} E_{n}^{\beta}, \quad \beta \in\right] \alpha_{j-1}^{(n)}, \alpha_{j}^{(n)}\left[, \quad j=1,2, \ldots, k_{n} \quad\left(\alpha_{0}^{(n)}=0\right)\right.
$$

are well defined. These numbers will be called critical multiplicities. We shall write them in the form

$$
N_{n}=\left(q_{1}^{(n)}, q_{2}^{(n)}, \ldots, q_{k_{n}}^{(n)}\right)
$$

It is clear that $q_{1}^{(n)}=n+1$ since $n+1$ is the number of extremal points in the real case; cf. Meinardus [8, p. 20]. We have, e.g.,

$$
\begin{gathered}
N_{1}=(2,1), \\
N_{2}=(3,2,3,2),
\end{gathered}
$$

(Geiger and Opfer [4]). We could call any soluble uniform approximation problem nice if it consists of the determination of $n$ real parameters and has the property that the error curve of a best approximation has $n+1$ extremal points. Under certain conditions such a problem could be dealt with by a Newton-type algorithm which, e.g., was suggested by Hettich [7]. In case there are less than $n+1$ extremal points (the usual case in nonlinear real approximation), another more difficult approach is needed, which again is described in the mentioned paper by Hettich. With this terminology we see that our problems are not necessarily nice. 
2. Description of the Numerical Method. The main idea is the reformulation of the original problem $\left\|T_{n}\right\|_{\infty}=\min$ as a semi-infinite linear optimization problem. This can be done by using the equation

$$
\begin{aligned}
D(r) & =\{z \in \mathbf{C}:|z| \leqslant r\} \\
& =\{z \in \mathbf{C}: \operatorname{Re} \bar{\gamma} z \leqslant r, \text { for all } \gamma \in \mathbf{C} \text { with }|\gamma|=1\},
\end{aligned}
$$

which means that the disk $D(r)$ is represented as the intersection of its supporting halfplanes.

More precisely we use the following

Lemma 2.1. Let $D \subset \mathrm{C}$ be a nonempty set, $f: D \rightarrow \mathrm{C}$ a function, and $r$ a nonnegative real number.

Define

$$
\begin{aligned}
& M_{1}=\{z:|f(z)| \leqslant r\} \\
& M_{2}=\{z: \operatorname{Re} \bar{\gamma} f(z) \leqslant r \text { for all } \gamma \in \mathbf{C} \text { with }|\gamma|=1\}
\end{aligned}
$$

Then

$$
M_{1}=M_{2} \text {. }
$$

Proof. Let $z \in M_{1}$. Then, for all $\gamma \in \mathbf{C}$ with $|\gamma|=1$, we have $\operatorname{Re} \bar{\gamma} f(z) \leqslant$ $|\bar{\gamma} f(z)|=|f(z)| \leqslant r$, and hence $z \in M_{2}$. Conversely, let $z \in M_{2}$. If $f(z)=0$, then $z \in M_{1}$. Assume therefore that $f(z) \neq 0$. In this case $\gamma=f(z) /|f(z)|$ is of modulus one, and hence $\operatorname{Re} \bar{\gamma} f(z)=|f(z)| \leqslant r$, hence $z \in M_{1}$.

Applied to the problem under investigation we obtain from Lemma 2.1 the semi-infinite linear optimization problem

$$
\left\{\begin{array}{l}
\operatorname{Re} \bar{\gamma} T_{n}(z) \leqslant a_{n} \text { for all } z \in \partial S^{\alpha} \text { and all } \gamma \in \mathbf{C} \text { with }|\gamma|=1, \\
a_{n}=\min .
\end{array}\right.
$$

If we set $\gamma=e^{i \phi}, \phi \in\left[0,2 \pi\left[\right.\right.$ and $T_{n}(z)=z^{n}+\sum_{j=0}^{n-1} a_{j} z^{j}$, then (2.2) becomes

$$
\left\{\begin{array}{l}
-\sum_{j=0}^{n-1} a_{j} g_{j}(z, \phi)+a_{n} \geqslant g_{n}(z, \phi), \\
\quad \text { for all } z \in \partial S^{\alpha} \text { and for all } \phi \in[0,2 \pi[,
\end{array}\right.
$$

where

$$
g_{j}(z, \phi)=\operatorname{Re}\left(z^{j}\right) \cos \phi+\operatorname{Im}\left(z^{j}\right) \sin \phi, \quad j=0, \ldots, n .
$$

For ease of computation, $z$ was represented in polar coordinates. The semi-infinite problem was solved approximately in a first step by admitting only finitely many $\phi \in\left[0,2 \pi\left[\right.\right.$ and finitely many $z \in \partial S^{\alpha}$. In this case (2.3) is an ordinary finite linear optimization problem which can be solved by a suitable version of the Simplex algorithm; cf. Glashoff and Gustafson [5, Chapter IV]. The result of this computation is an approximation for

(1) the coefficients $a_{0}, a_{1}, \ldots, a_{n-1}$ and the norm $a_{n}$ and

(2) for the error function

$$
f(z, \phi)=-\sum_{j=0}^{n-1} a_{j} g_{j}(z, \phi)+a_{n}-g_{n}(z, \phi)
$$


In particular, one obtains the number $q$ and the positions of the zeros of $f$ :

$$
f\left(z_{j}, \phi_{j}\right)=0, \quad j=1,2, \ldots, q .
$$

From solving the dual problem, one obtains the "masses"

$$
w_{1}, w_{2}, \ldots, w_{q}, \quad w_{j}>0, \quad \sum_{j=1}^{q} w_{j}=1 .
$$

Now, if we want to improve on these approximations, we regard the $n+1+4 q$ quantities $\left(z_{j} \in \mathbf{C}, j=1,2, \ldots, q\right)$

$$
a_{0}, a_{1}, \ldots, a_{n}, z_{1}, z_{2}, \ldots, z_{q}, \quad \phi_{1}, \phi_{2}, \ldots, \phi_{q}, \quad w_{1}, w_{2}, \ldots, w_{q}
$$

as unknowns in the following problem; cf. Glashoff and Gustafson [5, Section 16].

$$
\begin{gathered}
f\left(z_{j}, \phi_{j}\right)=0, \quad j=1,2, \ldots, q, \\
\sum_{k=1}^{q} w_{k} g_{j}\left(z_{k}, \phi_{k}\right)=0, \quad j=0,1, \ldots, n-1, \\
\sum_{k=1}^{q} w_{k}=1, \\
\left(z_{j}, \phi_{j}\right) \text { are local minima of } f, \quad j=1,2, \ldots, q .
\end{gathered}
$$

Condition (2.9) always yields $3 q$ equations either by setting partial derivatives to be zero or by boundary positions of $\left(z_{j}, \phi_{j}\right)$. Thus (2.6)-(2.9) results in a system of $n+1+4 q$ equations for the same number of real unknowns.

This system was solved using Newton's method, where the already computed approximations were taken as starting values. Since the $T_{n}^{\alpha}$ are symmetric (i.e., $\left.T_{n}^{\alpha}(\bar{z})=\overline{T_{n}^{\alpha}(z)}\right)$, one can restrict the domain to

$$
\left\{z \in \partial S^{\alpha}: \operatorname{Im} z \geqslant 0\right\},
$$

and consequently we obtain

$$
q=q(\alpha)=\# \text { ess } E_{n}^{\alpha} \text {. }
$$

If $\alpha \in A_{n}$, i.e., $\alpha$ is a critical angle and

$$
q(\alpha+\varepsilon)=q(\alpha-\varepsilon) \pm 1,
$$

for all sufficiently small and positive $\varepsilon$, then in (2.9) the number of local minima is reduced by one. That means that a certain "mass" $w_{k_{0}}$ vanishes. Since for $\alpha=0$ (real case) we always have $n+1$ positive masses, $\alpha=0$ cannot be a critical angle.

Therefore we can introduce $\alpha$ in (2.6)-(2.9) as a new unknown and simultaneously replace $w_{k_{0}}$ by zero, which leaves the total number of unknowns unchanged.

It should be noticed that a slightly different idea was used by Glashoff and Roleff [6] to obtain the same semi-infinite linear analogue of the Chebyshev approximation problem. However, the idea used here, namely to replace the disks in $\mathbf{C}$ by the union of its supporting halfplanes, immediately carries over to approximation problems with vector valued functions.

There is another investigation by Streit and Nuttall [10], who treat the linear complex approximation problem, in which $D$ is a priori discrete, by introducing a new variable as we did in (2.1). 
3. Numerical Figures. In Tables $2-7$ we list the coefficients $a_{j}^{(n)}, j=0,1, \ldots, n-1$, of $T_{n}^{\alpha}$ for $n=1(1) 6$ and $\alpha=0^{\circ}\left(5^{\circ}\right) n \cdot 180^{\circ} /(n+1)$. In Table 8 we list critical angles and corresponding multiplicities of $T_{n}^{\alpha}$ for $n=1(1) 6$.

Figure $n, n=1,2, \ldots, 6$, contains the positions of the (essential) extremal points of $T_{n}^{\alpha}$ for $\alpha=0^{\circ}, 5^{\circ}, 10^{\circ}, \ldots$ The vertical axis represents $\alpha$, the horizontal axis represents the distance $s$ from zero measured on the arc $\partial S^{\alpha}$. Thus a dot with coordinates $(s, \alpha)$ in Figure $n$ means that $T_{n}^{\alpha}$ has an extremal point on $\partial S^{\alpha}$ which has the shortest distance $s$ from $z=0$ measured on $\partial S^{\alpha}, n=1,2, \ldots, 6$. Figure 7 contains an enlarged detail of Figure 6.

Figure 8 shows the norm $\left\|T_{4}^{\alpha}\right\|$ in dependence of $\alpha$ and the corresponding coefficients also as they depend on $\alpha$.

From Figure 8 and the other given values of $\left\|T_{n}^{\alpha}\right\|$ it could be conjectured that $\left\|T_{n}^{\alpha}\right\|_{\infty}$ is differentiable with respect to $\alpha$. However, it can be shown that the $a_{j}^{(n)}$ are not differentiable with respect to $\alpha$. It suffices to look at the explicit expressions in Geiger and Opfer [4] for $n=1,2$.

For the case $n=4$ and $\alpha=\pi / 4$, we present in Figures 9 and 10 the corresponding lemniscate and the error curve. The lemniscate is defined by $\left\{z \in \mathrm{C}:\left|T_{4}^{\pi / 4}(z)\right|\right.$ $\left.=\left\|T_{4}^{\pi / 4}\right\|_{\infty}\right\}$ and the error curve by $\left\{w \in \mathrm{C}: w=T_{4}^{\pi / 4}(z), z \in \partial S^{\pi / 4}\right\}$.

TABLE 2

Norms and coefficients of $T_{1}^{\alpha}$

\begin{tabular}{r|cc}
$\alpha$ & $\left\|T_{\mathbf{1}}\right\|$ & $-a_{0}$ \\
\hline 0 & 0.500000 & 0.500000 \\
5 & 0.501910 & 0.501910 \\
10 & 0.507713 & 0.507713 \\
15 & 0.517638 & 0.517638 \\
20 & 0.532089 & 0.532089 \\
25 & 0.551689 & $0.5516,89$ \\
30 & 0.577350 & 0.577350 \\
35 & 0.610387 & 0.610387 \\
40 & 0.652704 & 0.652704 \\
45 & 0.707107 & 0.707107 \\
50 & 0.766044 & 0.642788 \\
55 & 0.819152 & 0.573576 \\
60 & 0.266025 & 0.500000 \\
65 & 0.906308 & 0.422618 \\
70 & 0.939693 & 0.342020 \\
75 & 0.965926 & 0.253819 \\
80 & 0.984809 & 0.1736 .48 \\
85 & 0.996 .195 & 0.087156 \\
90 & 1.000000 & 0.000000
\end{tabular}


TABLE 3

Norms and coefficients of $T_{2}^{\alpha}$

\begin{tabular}{r|ccc}
$\alpha$ & $\left\|T_{\mathbf{2}}\right\|$ & $\boldsymbol{a}_{\mathbf{0}}$ & $-\boldsymbol{a}_{\mathbf{1}}$ \\
\hline 0 & 0.125000 & 0.125000 & 1.000000 \\
5 & 0.131004 & 0.131004 & 1.019809 \\
10 & 0.153659 & 0.153659 & 1.091273 \\
15 & 0.205605 & 0.205605 & 1.164525 \\
20 & 0.254855 & 0.254855 & 1.179178 \\
25 & 0.297071 & 0.297071 & 1.175545 \\
30 & 0.333333 & 0.333333 & 1.154701 \\
35 & 0.364505 & 0.364505 & 1.117737 \\
40 & 0.391279 & 0.391279 & 1.065781 \\
45 & 0.414214 & 0.414214 & 1.000000 \\
50 & 0.437527 & 0.437527 & 1.000000 \\
55 & 0.465733 & 0.465733 & 1.000000 \\
60 & 0.500000 & 0.500000 & 1.000000 \\
65 & 0.541936 & 0.541936 & 1.000000 \\
70 & 0.593810 & 0.593810 & 1.000000 \\
75 & 0.755775 & 0.586707 & 0.930931 \\
80 & 0.716881 & 0.532089 & 0.815207 \\
85 & 0.774798 & 0.474555 & 0.699757 \\
90 & 0.328427 & 0.414214 & 0.585786 \\
95 & 0.876672 & 0.351180 & 0.474508 \\
100 & 0.918447 & 0.285575 & 0.367128 \\
105 & 0.952684 & 0.217523 & 0.264839 \\
110 & 0.978346 & 0.147153 & 0.168807 \\
115 & 0.994435 & 0.074599 & 0.020164 \\
120 & 1.000000 & 0.000000 & 0.000000
\end{tabular}

TABLE 4

Norms and coefficients of $T_{3}^{\alpha}$

\begin{tabular}{|c|c|c|c|c|}
\hline$\alpha$ & $\left\|T_{3}\right\|$ & $-a_{0}$ & $a_{1}$ & $-a_{2}$ \\
\hline & & 0.03 & & 1.50 \\
\hline & 0.038243 & .038243 & 0.638574 & 1. \\
\hline 10 & 0.056051 & 0.056051 & 0.788605 & \\
\hline 15 & 0.069168 & $0.0691 \mathrm{kB}$ & 0.862537 & 3742 \\
\hline 20 & 0.081289 & 0.081289 & 0.919433 & 1.756856 \\
\hline 2.5 & 0.096515 & 0.096 .515 & 0.987671 & 1.794540 \\
\hline 30 & 0.118714 & 0.118714 & 1.079 & 1.841777 \\
\hline 35 & 0.150067 & 0.150067 & $1.16^{7}$ & 1.867046 \\
\hline 40 & 0.184793 & 0.184793 & 1.184793 & $1.81520^{7}$ \\
\hline 45 & 0.217545 & 0.819545 & 1.191667 & 1.752576 \\
\hline 50 & 0.253540 & 0.25 & & 1.678990 \\
\hline 55 & 0.286168 & 168 & & 94632 \\
\hline 60 & 0.316987 & 0.316 & 1.1 & \\
\hline 65 & $0.347^{1} 34$ & 0.347734 & 2974 & 1.440616 \\
\hline 70 & 0.3830 .38 & 0.38 & 724 & 008 \\
\hline 75 & 0.424426 & 0.424426 & 141694 & 559 \\
\hline 80 & 0.473814 & 0.473914 & 121 & 5515 \\
\hline 85 & 0.533875 & 0.533479 & 1700 & 1.243705 \\
\hline 90 & 0.600566 & 0.485968 & 1.000 & 1.086434 \\
\hline 95 & 0.6 .66 & $0.4370 ? 7$ & 0.86 & 0.932 .030 \\
\hline 100 & 0.731114 & 0.337020 & 0.736674 & 0.782117 \\
\hline 105 & 0.792163 & $0.3359,7 ?$ & 0.613881 & 0.6 .39341 \\
\hline 110 & 0.848395 & 0.28356 .4 & 0.497240 & 0.502367 \\
\hline 115 & 0.898232 & $0.23002 ?$ & 0.386 .719 & 0.375881 \\
\hline 12.0 & 0.9400 .35 & 0.175140 & 0.2921 .30 & 0.260599 \\
\hline 125 & 0.972113 & 0.118706 & 0.183145 & 0.158284 \\
\hline 130 & 0.992711 & 0.050451 & $0.08930 ?$ & 0.070769 \\
\hline & 1.000000 & 0.0000 ก & 0.000000 & 0.000000 \\
\hline
\end{tabular}


TABLE 5

Norms and coefficients of $T_{4}^{\alpha}$

\begin{tabular}{|c|c|c|c|c|c|}
\hline$\alpha$ & $\left\|T_{4}\right\|$ & $a_{0}$ & $-a_{1}$ & $a_{2}$ & $-a_{3}$ \\
\hline & & & & & \\
\hline & & & & & \\
\hline 10 & & & $.40 t$ & & \\
\hline 15 & & & 0.48 & & \\
\hline 20 & & & & & \\
\hline 25 & & & 0.68 & & \\
\hline 30 & & & & & \\
\hline 35 & 0.06 & & 0.81 & & \\
\hline 40 & & & 0.89 & & \\
\hline 45 & & & 0.97 & & \\
\hline 50 & & & 1.0 & & \\
\hline 55 & & & & & \\
\hline 60 & 0. & & & & \\
\hline 65 & 0. & & & & \\
\hline 70 & & & & & \\
\hline 75 & & & & & \\
\hline 80 & & & & & \\
\hline 8 & & & & & \\
\hline 90 & & & & & \\
\hline & & & & & \\
\hline 00 & & & & & \\
\hline & & & & & \\
\hline 110 & & & & & \\
\hline 115 & & 0.2 & 0.5 & & 0. \\
\hline 12 & & & & & \\
\hline 12 & & & & 0.4 & \\
\hline & & & & & \\
\hline 135 & & & & & \\
\hline 140 & & 0.040150 & 0.066728 & 0.070330 & 049562 \\
\hline & & & & & \\
\hline
\end{tabular}


TABLE 6

Norms and coefficients of $T_{5}^{\alpha}$

\begin{tabular}{|c|c|c|c|c|c|c|}
\hline$\alpha$ & $\left\|T_{5}\right\|$ & $-a_{0}$ & $a_{1}$ & $-a_{2}$ & $a_{3}$ & $-a_{4}$ \\
\hline 0 & & & & & & \\
\hline 5 & & & & & & \\
\hline 10 & & 0.004 & & & & \\
\hline 15 & & 0.007 & 0.255431 & 1.48 & & \\
\hline 20 & & & 0.318 & & & \\
\hline & & & & & & \\
\hline 30 & & $0.0 ? 1$ & 0.46 & 2.0 & & \\
\hline & & 0.029 & & 2.8 & & \\
\hline 40 & & & 0.6 & & & \\
\hline 45 & & 0.05 & 0.60 & & & \\
\hline & & 0.0 & 0.77 & & & \\
\hline & & 0.0 & 0.84 & & & \\
\hline & & 0.1 & & & & \\
\hline & & 0.1 & & & & \\
\hline & 91 & 0.16 & 1.0 & & & \\
\hline & & 0.1 & & & & \\
\hline & & & & & & \\
\hline & & 0.2 & & & & \\
\hline & & 0.3 & & & & \\
\hline 95 & & & & & & \\
\hline & & 0.3 & & & & \\
\hline & & & & & & \\
\hline & & 0.3 & & $1 . ?$ & & \\
\hline & & 0.283 & & & & \\
\hline & & & & & & \\
\hline & & & & & & \\
\hline & & & & & & \\
\hline & & 0.122422 & 0.23 & & & \\
\hline & & & & 0.1 & 98 & \\
\hline & & & & & & \\
\hline & & & & 0.000000 & 0.000000 & \\
\hline
\end{tabular}


TABLE 7

Norms and coefficients of $T_{6}^{\alpha}$

\begin{tabular}{|c|c|c|c|c|c|c|c|}
\hline$\alpha$ & $\left\|T_{6}\right\|$ & $a_{0}$ & $-a_{1}$ & $a_{2}$ & $-a_{3}$ & $a_{4}$ & $-a_{5}$ \\
\hline $\begin{array}{l}0 \\
5\end{array}$ & & & & & & & \\
\hline $10^{5}$ & 519 & 0.001519 & 0.084967 & 0.792396 & 2.772877 & & \\
\hline 15 & & 0.002476 & 0.119685 & & 3.203455 & & \\
\hline 20 & $.00^{\circ}$ & 0.003 & 0.16 & 1.225248 & & & \\
\hline 25 & 0.0 & 0.006 & 0.21 & & 4.008 & & \\
\hline & & 0.009 & 0.27 & 1.56 & 4.2 & & \\
\hline & .013 & 0.013260 & 0.33 & 1.85 & & & 68 \\
\hline 40 & 0.01 & 0.018 & 0.40 & 2.05 & 4.68 & & \\
\hline 45 & 0.02 & 0.026 & 0.47 & 2.21 & 4.78 & & 738 \\
\hline & 0.03 & 0.03 & 0.5 & 73 & 2 & & \\
\hline & & 0.048 & 0.62 & & & & \\
\hline & 0.0 & 0.063 & 0.70 & & & & 3. \\
\hline 65 & 0 & 0.0 & 0.7 & & & & \\
\hline & & $0.1 \mathrm{r}$ & & & & & \\
\hline 75 & 0 & 0.1 & 0.92 & 2.58 & 4.1 & & 16 \\
\hline 80 & 1 & 0.1 & 0.9 & & & & \\
\hline 85 & & & $1 . c$ & & 3.6 & & \\
\hline 90 & 0 & 0.23 & $1 . c$ & & & & \\
\hline 95 & 0 & 0.26 & 1.0 & & & & \\
\hline & & & 1.01 & & 2.6 & & \\
\hline 105 & 0.4 & 0.3 & 0.99 & & & & \\
\hline & & & 0.9 & & & & \\
\hline & 0.5 & 0.2 & 0.7 & & & & \\
\hline & & & 0.6 & & & & \\
\hline 125 & 0 & 0.2 & 0.40 & 0.76 & 0.9 & & \\
\hline & .785 & 0.172084 & 0.38 & & 0.6 & & \\
\hline & & & 0.28 & 0.4148 & 0.4 & & \\
\hline & & 0.101 & 0.201857 & 0.2820 & 0.32 & 960 & \\
\hline & & & & 0.17 & & & \\
\hline & & & & & & & \\
\hline & 1.0 & 0000 & 0.000000 & .000000 & 0.000000 & .000000 & 0000 \\
\hline
\end{tabular}


TABLE 8

Critical angles $\alpha_{j}$ (in degree) and multiplicities

(in parentheses) of $T_{n}^{\alpha}$ for $n=1(1) 6$

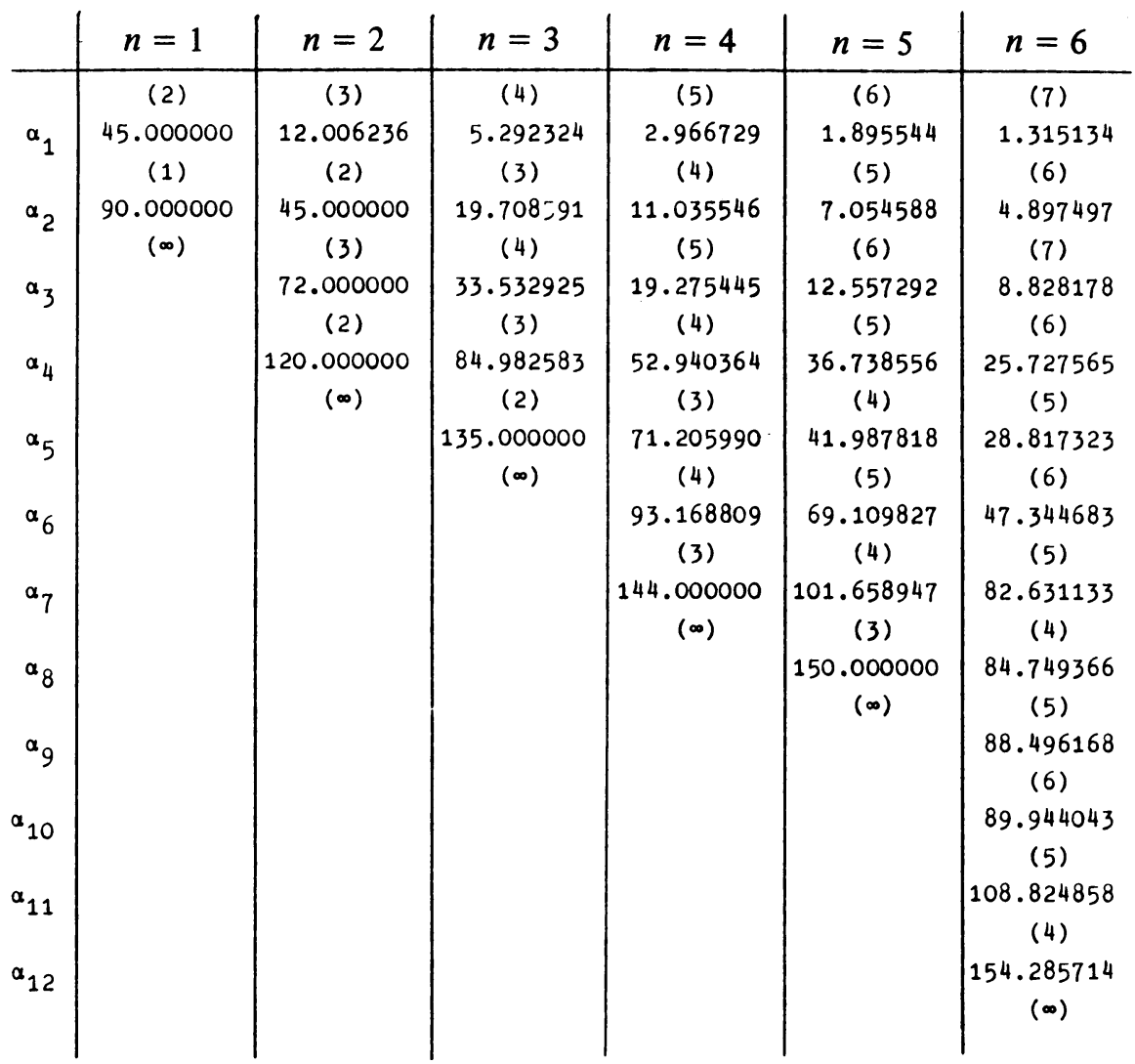




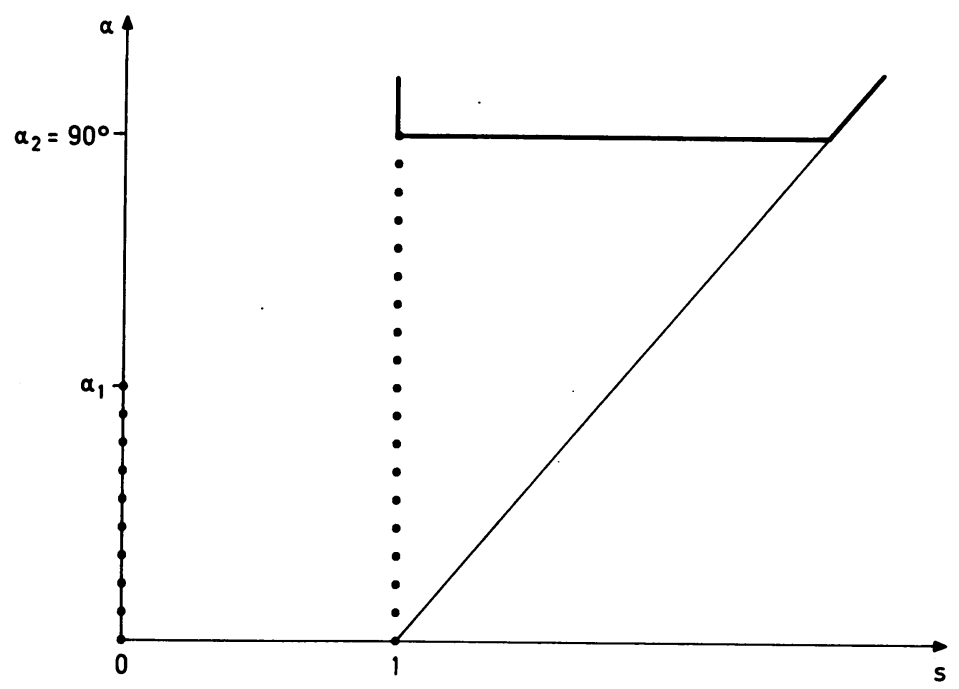

FIGURE 1

Positions of extremal points of $T_{1}^{\alpha}$

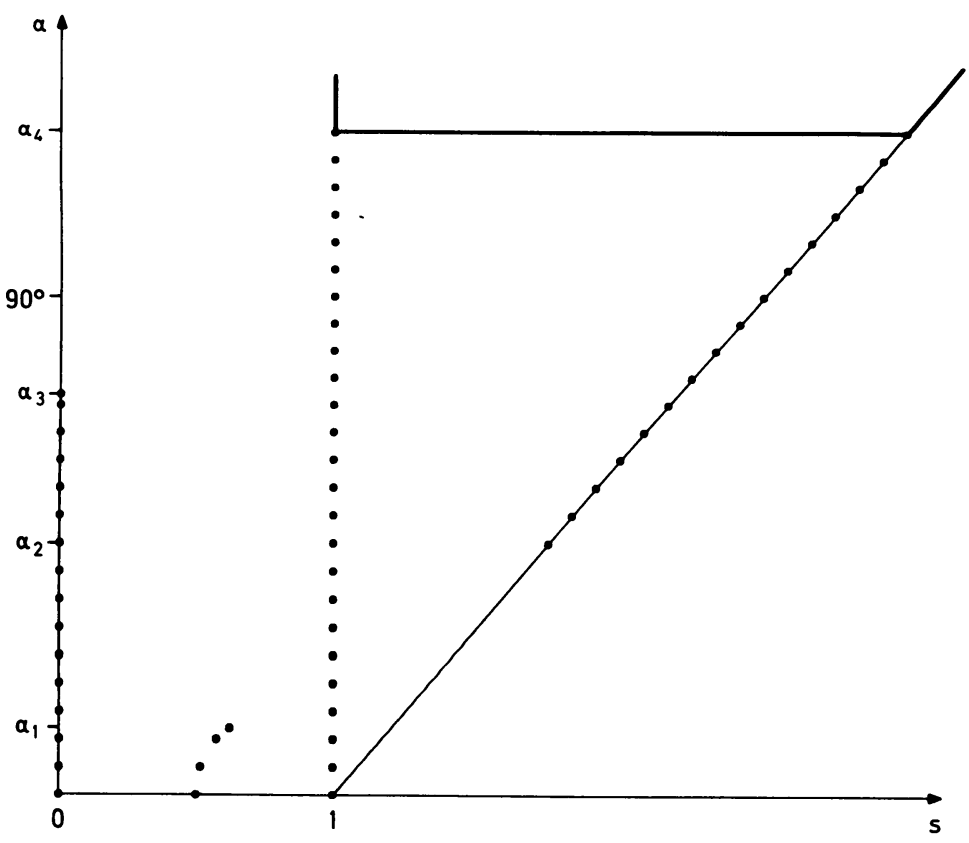

FIGURE 2

Positions of extremal points of $T_{2}^{\alpha}$ 


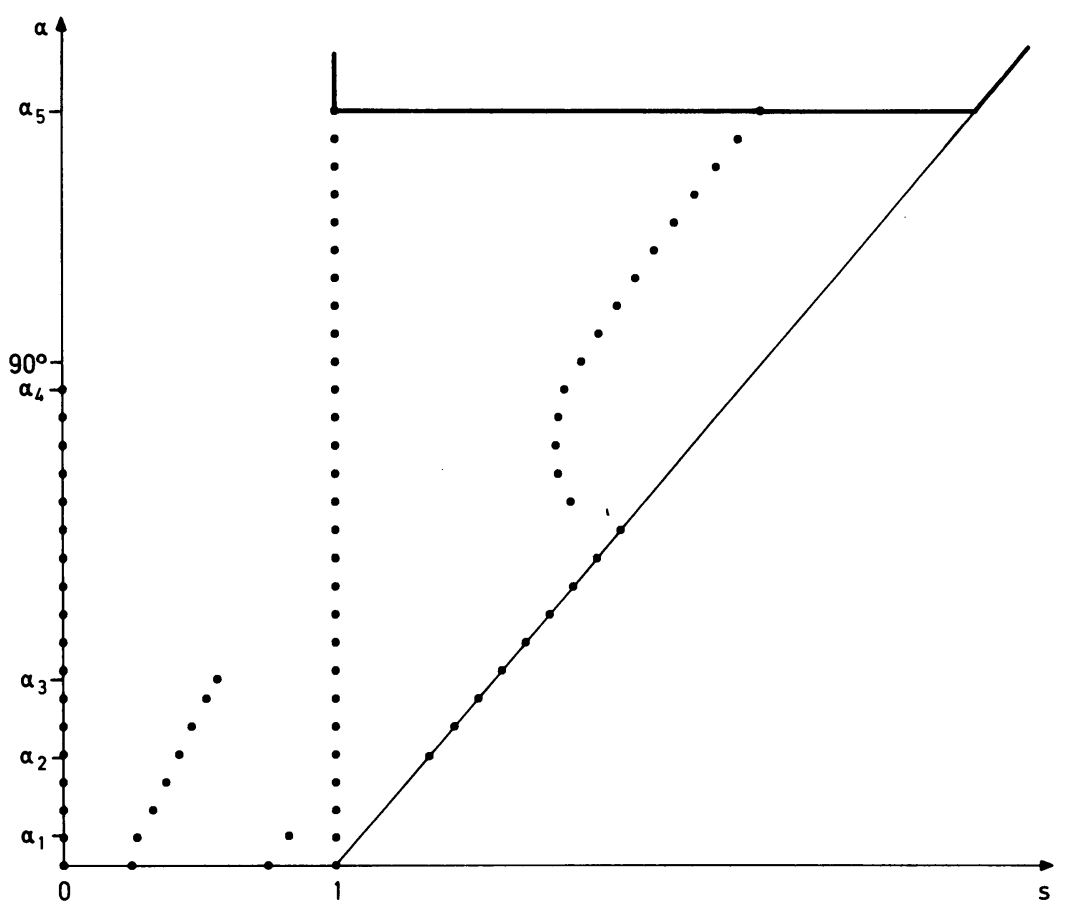

FIGURE 3

Positions of extremal points of $T_{3}^{\alpha}$

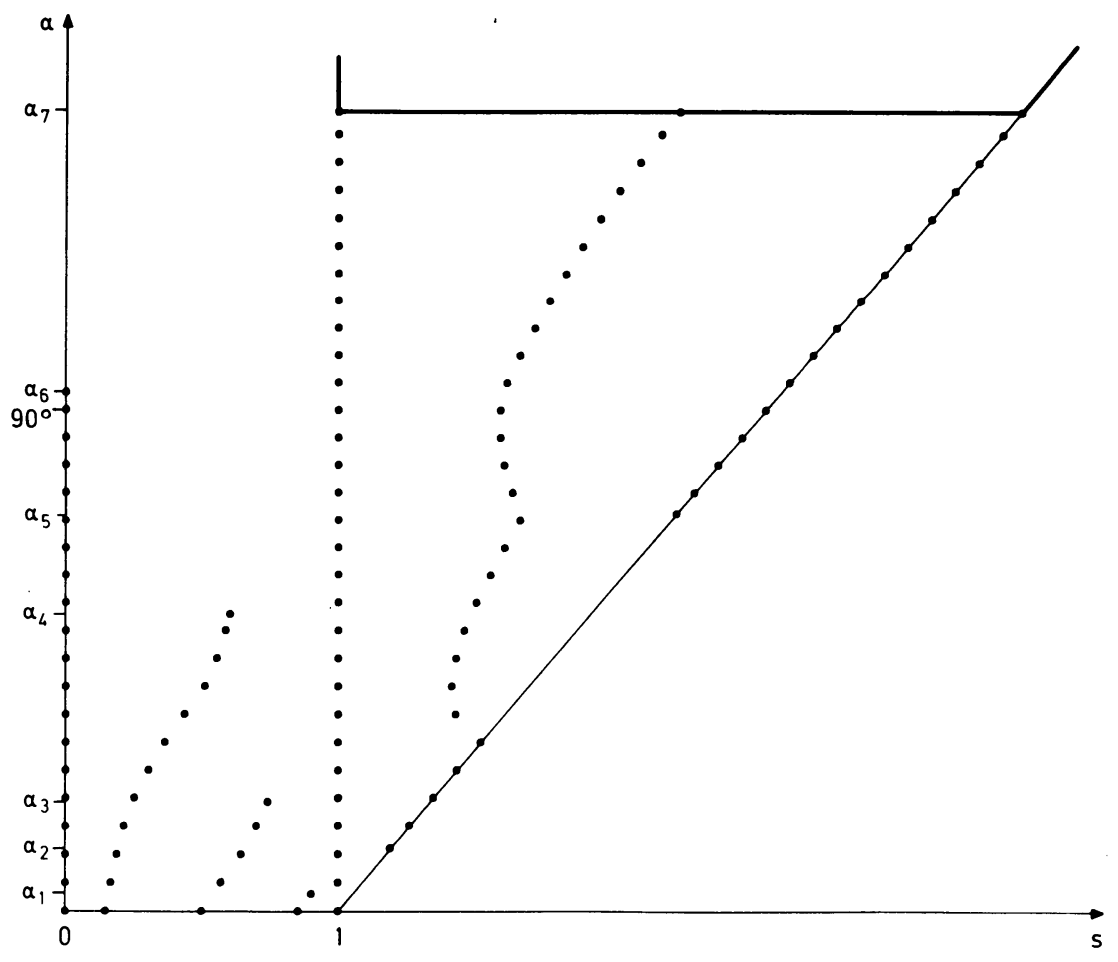

FIGURE 4

Positions of extremal points of $T_{4}^{\alpha}$ 


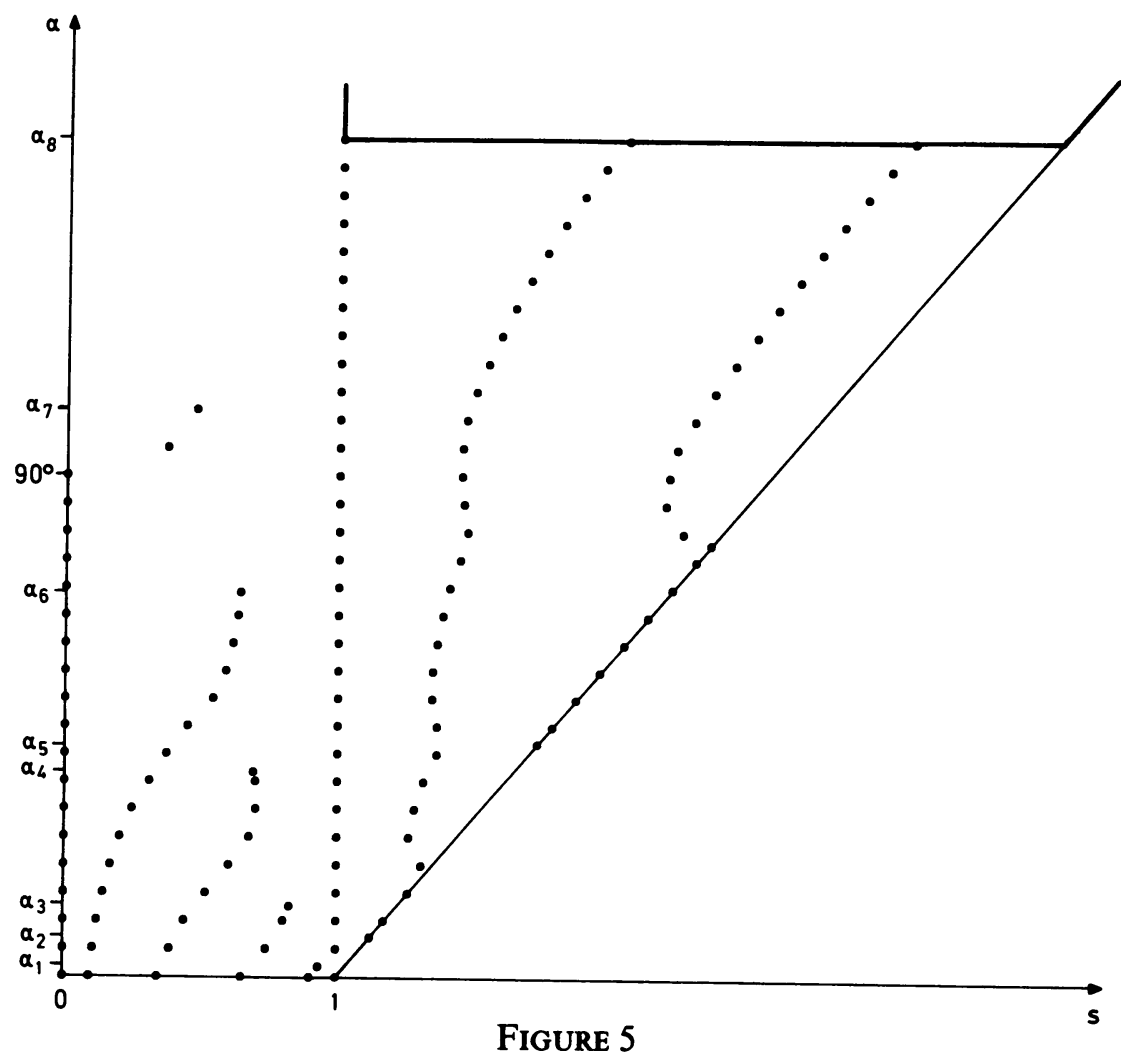

Positions of extremal points of $T_{5}^{\alpha}$

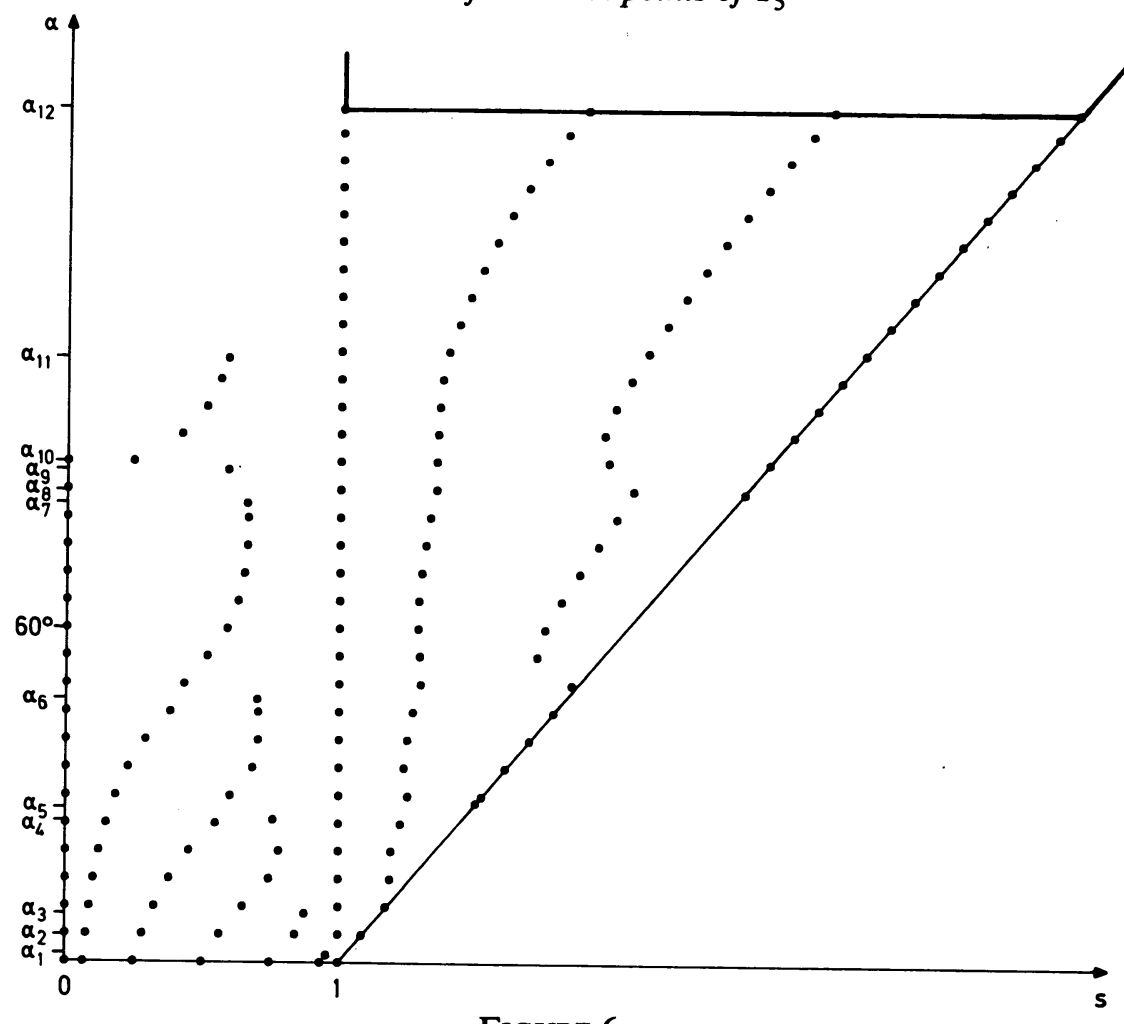

Figure 6

Positions of extremal points of $T_{6}^{\alpha}$ 
COMPLEX CHEBYSHEV POLYNOMIALS

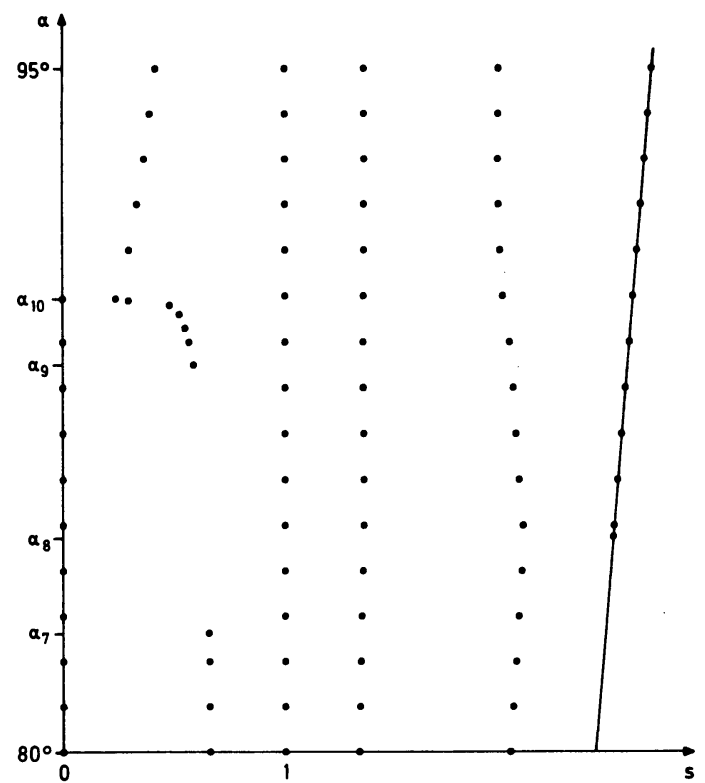

Figure 7

Enlarged detail of Figure 6 near $\alpha=90^{\circ}$

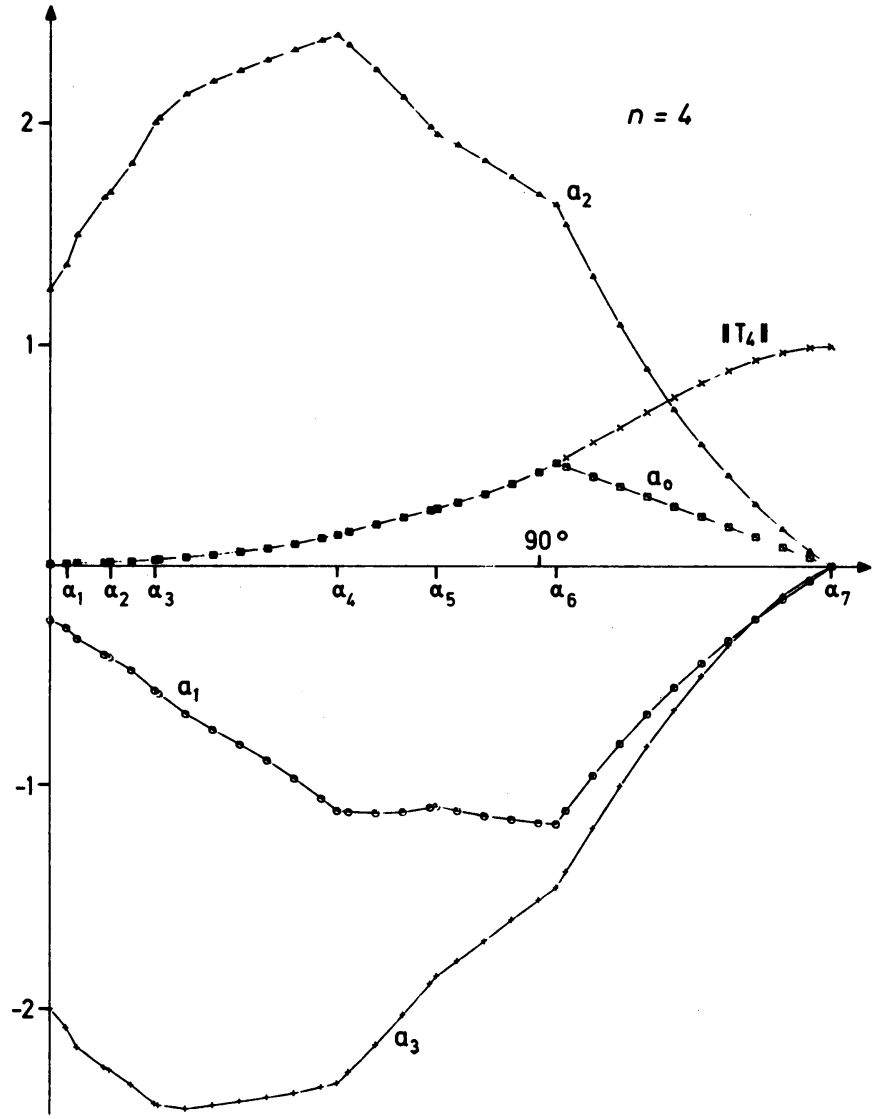

FIGURE 8

$\left\|T_{4}^{\alpha}\right\|_{\infty}, a_{j}^{(4)}, j=0(1) 3$ as functions of $\alpha \in\left[0^{\circ}, 144^{\circ}\right]$ 


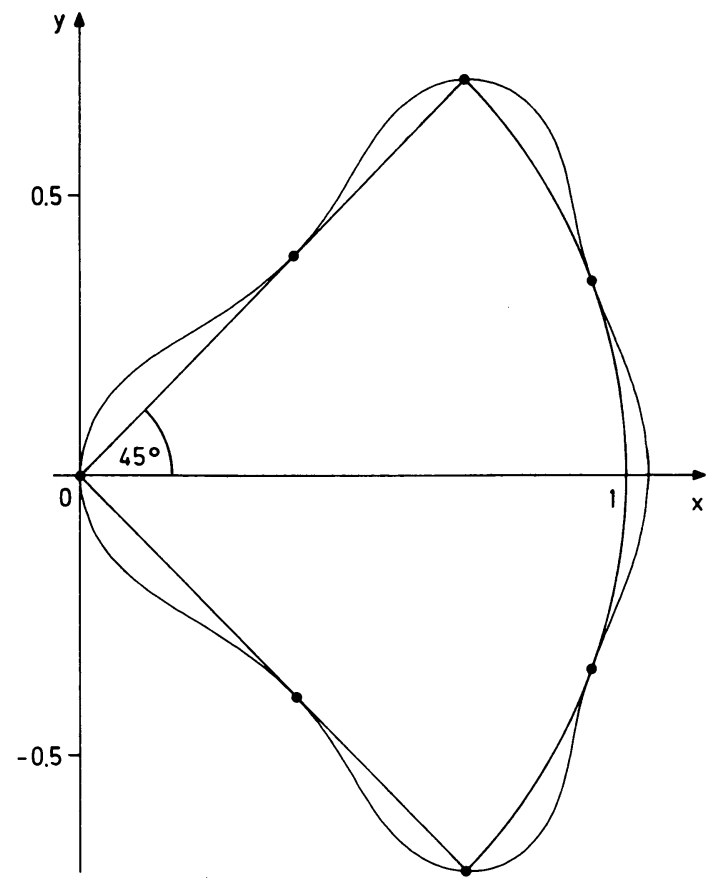

FigURE 9

Lemniscate $\left|T_{4}^{\pi / 4}(z)\right|=\left\|T_{4}^{\pi / 4}\right\|_{\infty}$

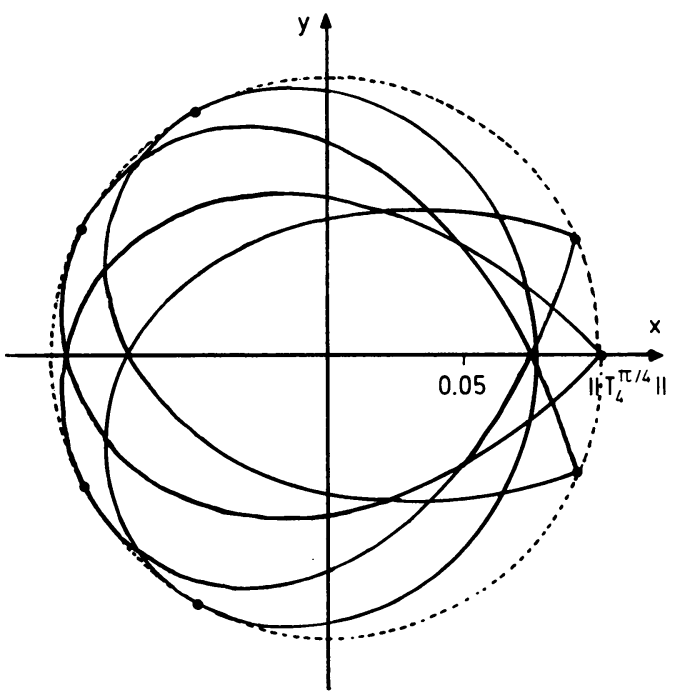

Figure 10

Error curve $w=T_{4}^{\pi / 4}(z), z \in \partial S^{\pi / 4}$

Institute for Applied Mathematics

Bundesstrasse 55

D-2000 Hamburg 13, West Germany 
1. M. Abramowitz \& I. A. Stegun (Editors), Handbook of Mathematical Functions, fifth printing, Dover, New York, 1968.

2. J. P. Coleman \& A. J. Monaghan, Chebyshev Expansions for the Bessel Function $J_{n}(z)$ in the Complex Plane, University of Durham, 1980. (Preprint.)

3. G. H. Elliot, Polynomial Approximation in the Complex Plane Using Generalised Humbert Polynomials, Lecture at Dundee Biennial Conference on Numerical Analysis, 1981.

4. C. Geiger \& G. Opfer, "Complex Chebyshev polynomials on circular sectors," J. Approx. Theory, v. 24, 1978, pp. 93-118.

5. K. Glashoff \& S. Å. Gustafson, Einführung in die lineare Optimierung, Wissenschaftliche Buchgesellschaft, Darmstadt, 1978.

6. K. Glashoff \& K. RolefF, "A new method for Chebyshev approximation of complex-valued functions," Math. Comp., v. 36, 1981, pp. 233-239.

7. R. HetтісH, "Numerical methods for nonlinear Chebyshev approximation," in Approximation in Theorie und Praxis (G. Meinardus, Ed.), Bibliographisches Institut Mannheim, Wien, Zürich, 1979, pp. $139-156$.

8. G. MeInARdus, Approximation of Functions: Theory and Numerical Methods, Springer-Verlag, Berlin, Heidelberg and New York, 1967.

9. G. OPFER, "An algorithm for the construction of best approximations based on Kolmogorov's criterion," J. Approx. Theory, v. 23, 1978, pp. 299-317.

10. R. L. Streit \& A. H. Nuttall, Linear Chebyshev Complex Function Approximation, Technical Report 6403, Naval Underwater Systems Center, Newport, R. I., New London, Conn., 1981. 